\title{
Redistributing Income under Proportional
}

\section{Representation: A Correction*}

\author{
Massimo Morelli \\ Bocconi University, IGIER and CEPR, massimo.morelli@unibocconi.it
}

Margherita Negri

University of St Andrews, mn48@st-andrews.ac.uk

*We thank the editor Emir Kamenica and two anonymous referees for their useful comments. 


\begin{abstract}
Austen-Smith (2000) reports a theoretical result that if the cost of entering the workforce is sufficiently low, winner-take-all political systems induce endogenous redistribution levels that are systematically lower than those determined by proportional representation systems (Proposition 6). The proof in Austen-Smith (2000) has a mistake. We explain the mistake and offer an alternative proof. The conclusion of the original paper continues to hold.
\end{abstract}


Austen-Smith (2000) reports a theoretical result that if the cost of entering the workforce is sufficiently low, winner-take-all political systems induce endogenous redistribution levels that are systematically lower than those determined by proportional representation systems (Proposition 6). The proof in Austen-Smith (2000) has a mistake. We explain the mistake and offer an alternative proof. The conclusion of the original paper continues to hold.

\section{The problem}

This section explains the mistake in the original paper. Before doing that, we review the modelling assumptions and results that are affected by the mistake. We refer to the original paper for a careful and exhaustive description of the model and do not report it here.

The main result of the paper (Proposition 6) shows that winner-take-all systems are associated to lower redistribution than proportional representation ones. The tax rate im-

plemented under winner-take-all systems, $t_{l}\left(\theta_{m}\right)$, is assumed to be the one preferred by the median type $\theta_{m}$ (under the assumption that this type is an employee). Under proportional representation systems, the tax rate is decided through a legislative bargaining process. The implemented rate, $t_{\mathcal{L}}$, is the one maximizing the utility of the average employee. More precisely, $t_{\mathcal{L}}$ is the only PRPE-stable level of taxation (i.e. the only tax level such that, if the status quo is $t_{0}=t_{\mathcal{L}}$, the legislative bargaining process will select $t_{\mathcal{L}}$ as an outcome). This is shown in Proposition 4. The proof of the proposition is a direct consequence of Lemma 3. In turn, the lemma relies on the conclusion that $t_{\mathcal{E}}<t_{\mathcal{L}}<t_{\mathcal{D}}$, where $t_{\mathcal{E}}$ and $t_{\mathcal{D}}$ are the tax levels maximizing the average employers' and unemployed individuals' utilities, respectively.

In order to obtain well defined tax levels, the paper relies on single-peakedness of individuals' preferences. In particular, Lemma 2 and Proposition 2 show that employees' and unemployed individuals' net income is strictly concave in $t$, while employers' net income is strictly quasi-concave in the tax level. Furthermore, Proposition 2 establishes that $t_{e}(\theta) \leq t_{l}(\theta) \leq t_{d}(\theta)$ (where $t_{j}(\theta)$ denotes the optimal tax rate for a type $\theta$ in occupation 
$j \in\{e, l, d\})$ and that, for each occupation, the preferred tax level is (weakly) decreasing in type. This has two important implications for the results in the paper: first, it allows one to apply the median voter theorem and conclude that the implemented tax rate under winner-take-all systems is $t_{l}\left(\theta_{m}\right)$. Secondly, it implies that $t_{\mathcal{E}}<t_{\mathcal{L}}<t_{\mathcal{D}}$, opening the way to the results for proportional representation.

The argument showing quasi-concavity of employers' income, however, contains a mistake. Let $y_{e}(\cdot, \theta)$ denote the gross income of an employer of type $\theta$. The second derivative of employers' net income with respect to the tax rate $t$ is

$$
\begin{aligned}
\frac{d^{2} x_{e}(t, \theta)}{d t^{2}} & =b^{\prime \prime}(t)-(1-t) y_{e}^{\prime \prime}(\cdot, \theta)+2 y_{e}^{\prime}(\cdot, \theta) \\
& =b^{\prime \prime}(t)-(1-t)\left[\left(w_{t}^{*}\right)^{2} L_{w}\left(w^{*}, \theta\right)+w_{t t}^{*} L\left(w^{*}, \theta\right)\right]+2 w_{t}^{*} L\left(w^{*}, \theta\right)
\end{aligned}
$$

The first term is the second derivative of the benefits with respect to $t$ and is always negative (Lemma 2). In the second and third terms of the last line, $w_{t}^{*}$ and $w_{t t}^{*}$ are the first and second derivatives of the equilibrium wage with respect to $t, L\left(w^{*}, \theta\right)$ is the optimal amount of labor demanded by an employer of type $\theta$ and $L_{w}\left(w^{*}, \theta\right)$ is its derivative with respect to the wage rate. The author shows that

$$
\lim _{\theta \downarrow 0} \frac{d^{2} x_{e}(t, \theta)}{d t^{2}}=b^{\prime \prime}(t)<0
$$

Then, proving that

$$
\frac{d}{d \theta}\left[\frac{d^{2} x_{e}(t, \theta)}{d t^{2}}\right] \leq 0
$$

is enough to obtain the result. This third derivative can be obtained by directly differentiating $d^{2} x_{e}(t, \theta) / d t^{2}$ with respect to $\theta$ or, as done in the paper, by differentiating $d^{2} x_{e}(t, \theta) / d t d \theta$ with respect to $t$ and using Young's theorem. We have

$$
\frac{d^{2} x_{e}(t, \theta)}{d t d \theta}=-(1-t)\left(w_{t}^{*}\right)^{2} L_{\theta}\left(w^{*}, \theta\right)-F_{\theta}\left(L\left(w^{*}, \theta\right), \theta\right)<0
$$

where $F(L, \theta)$ is the production function of an employer of type $\theta$ that employees $L$ units of 
labor and $F_{\theta}(\cdot)$ represents its derivative with respect to the second argument. The author writes

$$
\frac{d}{d t}\left[\frac{d^{2} x_{e}(t, \theta)}{d t d \theta}\right]=-L_{\theta}\left(w^{*}, \theta\right)\left[2 w_{t}^{*}+(1-t) w_{t t}^{*}\right]-(1-t)\left(w_{t}^{*}\right)^{2} L_{\theta w}\left(w^{*}, \theta\right)
$$

The assumptions made on the production function ensure that $L_{\theta w}\left(w^{*}, \theta\right)>0$. Denoting by $\tilde{\epsilon}(t)$ the tax elasticity of the marginal equilibrium wage rate,

$$
\tilde{\epsilon}(t)=\frac{t}{w_{t}^{*}} w_{t t}^{*}
$$

the author assumes (first inequality in condition (5) in the paper)

$$
-2 t \leq(1-t) \tilde{\epsilon}(t)
$$

This is equivalent to assuming that the term in square brackets in (1) is positive. Since $L\left(w^{*}, \theta\right)$ is increasing in $\theta$, condition (2) is enough to ensure that (1) is negative. Equation (1), however, is wrong. The correct version should be

$$
\frac{d}{d t}\left[\frac{d^{2} x_{e}(t, \theta)}{d t d \theta}\right]=L_{\theta}\left(w^{*}, \theta\right)\left[2 w_{t}^{*}-(1-t) w_{t t}^{*}\right]-(1-t)\left(w_{t}^{*}\right)^{2} L_{\theta w}\left(w^{*}, \theta\right)
$$

This implies that condition (2) cannot be used to establish the result, leaving the question of quasi-concavity unanswered.

In sum, the mistake implies that single-peakedness does not necessarily hold, and hence the results based on median voter utilities can be questioned. Fortunately, we now show that there is an alternative way to justify using median voter utilities. 


\section{An Alternative Proof}

In this section, we provide an alternative proof of the results. In the following lemma, we show that agents' preferences satisfy a weaker version of the single-crossing property (Gans and Smart (1996)). As in the original paper, let $\xi(t, \theta)$ denote a type $\theta$ 's maximum consumption level conditional on a tax rate $t$ and sorting equilibrium $w^{*}=w^{*}(t)$.

Lemma 1. For any two tax levels $t, t^{\prime}$ such that $t<t^{\prime}$,

1. $\xi\left(t, \theta_{m}\right) \geq \xi\left(t^{\prime}, \theta_{m}\right) \Rightarrow \xi(t, \theta) \geq \xi\left(t^{\prime}, \theta\right)$ for all $\theta>\theta_{m}$

2. $\xi\left(t^{\prime}, \theta_{m}\right) \geq \xi\left(t, \theta_{m}\right) \Rightarrow \xi\left(t^{\prime}, \theta\right) \geq \xi(t, \theta)$ for all $\theta<\theta_{m}$

Proof. Let $\theta>\theta_{m}$ first. A sufficient condition for 1 . to hold is

$$
\xi(t, \theta)-\xi\left(t^{\prime}, \theta\right) \geq \xi\left(t, \theta_{m}\right)-\xi\left(t^{\prime}, \theta_{m}\right)
$$

for all $t<t^{\prime}$. Rearranging terms, we get

$$
\xi(t, \theta)-\xi\left(t, \theta_{m}\right) \geq \xi\left(t^{\prime}, \theta\right)-\xi\left(t^{\prime}, \theta_{m}\right)
$$

Thus, 1. holds if the function $\Delta \xi(t) \equiv \xi(t, \theta)-\xi\left(t, \theta_{m}\right)$ is decreasing in $t$. By assumption, the median type is an employee, so that $\xi\left(t, \theta_{m}\right)=x_{l}\left(t, \theta_{m}\right)$. For all $\theta \in\left(\theta_{m}, \theta_{2}\left(t, w^{*}\right)\right)$, $\xi(t, \theta)=x_{l}(t, \theta)$. Then, $\Delta \xi(t)=(1-t)\left(\theta-\theta_{m}\right) w^{*}$. Differentiating it with respect to $t$ and rearranging terms, we get

$$
\frac{d \Delta \xi(t)}{d t}=-\left(\theta-\theta_{m}\right) w^{*} V(t)
$$

where

$$
V(t) \equiv 1-\frac{(1-t)}{w^{*}} \frac{d w^{*}}{d t}>0
$$

as shown in the proof of Lemma 2 in the original paper. For all $\theta \in\left[\theta_{2}, \bar{\theta}\right), \xi(t, \theta)=x_{e}(t, \theta)$. 
Then, $\Delta \xi(t)=(1-t)\left[y_{e}(t, \theta)-w^{*} \theta_{m}\right]$ and

$$
\frac{d \Delta \xi(t)}{d t}=-\left[y_{e}(t, \theta)-w^{*} \theta_{m}\right]+(1-t)\left[\frac{d y_{e}(t, \theta)}{d t}-\theta_{m} \frac{d w^{*}}{d t}\right]
$$

By the envelope theorem,

$$
\frac{d y_{e}(t, \theta)}{d t}=-L\left(w^{*}, \theta\right) \frac{d w^{*}}{d t}
$$

Then,

$$
\frac{d \Delta \xi(t)}{d t}=-\left[y_{e}(t, \theta)-w^{*} \theta_{m}\right]-(1-t)\left[L\left(w^{*}, \theta\right)+\theta_{m}\right] \frac{d w^{*}}{d t}<0
$$

as gross income is increasing in $\theta$ and wage is increasing in $t$.

By a similar reasoning, a sufficient condition for 2 . to hold is $d \Delta \xi(t) / d t>0$, whenever $\theta<\theta_{m}$. For all $\theta \in\left[\theta_{1}\left(t, w^{*}\right), \theta_{m}\right), \xi(t, \theta)=x_{l}(t, \theta)$ and $\Delta \xi(t)=(1-t)\left(\theta-\theta_{m}\right) w^{*}$. For all types $\theta \in\left(0, \theta_{1}\left(t, w^{*}\right)\right), \xi(t, \theta)=x_{d}(t, \theta)$ and $\Delta \xi(t)=-(1-t) \theta_{m} w^{*}+c$. In both cases, $V(t)>0$ implies $d \Delta \xi(t) / d t>0$.

By Lemma 1, the implemented tax level under the winner-take-all system will be the one preferred by the median voter, $t_{l}\left(\theta_{m}\right)$.

Consider the legislative bargaining process taking place under the proportional representation system. The existence of a $t_{\mathcal{E}}$ maximizing the average employers' utility is ensured by the fact that the utility function is continuous and $t \in[0,1]$. The problem is that, without single-peakedness, one cannot establish that $t_{\mathcal{E}}$ is i) unique and ii) such that $t_{\mathcal{E}}<t_{\mathcal{L}}<t_{\mathcal{D}}$. As a matter of fact, this is not necessary to prove the main result. Whenever, the party of the employers proposes $t_{\mathcal{E}}$ such that $t_{\mathcal{L}}<t_{\mathcal{E}}<t_{\mathcal{D}}$, Lemma 3 and Proposition 4 imply that the only PRPE-stable level of taxation is $t_{\mathcal{E}}$. Then, Proposition 6 ensures that $t_{l}\left(\theta_{m}\right)<t_{\mathcal{L}}<t_{\mathcal{E}}$. An identical reasoning holds for the case in which $t_{\mathcal{L}}<t_{\mathcal{D}}<t_{\mathcal{E}}$. Then, the tax level implemented under proportional representation systems will still be higher than the one implemented under winner-take-all.

As a final remark, notice that, given our alternative proof of the results, the lower bound 
of assumption (5) in the paper is no longer necessary. ${ }^{1}$

\section{References}

Austen-Smith, D. 2000. "Redistributing income under proportional representation". Journal of Political Economy 108 (December): 1235-1269.

Gans, J. S. and M. Smart 1996. "Majority voting with single-crossing preferences". Journal of Public Economics 59 (February): 219-237.

\footnotetext{
${ }^{1}$ We thank an anonymous referee for pointing this out.
} 